\title{
Laboratory observations of double-diffusive convection using high-frequency broadband acoustics
}

\author{
Tetjana Ross · Andone Lavery
}

Received: 29 July 2008 / Revised: 2 September 2008 / Accepted: 3 September 2008

\begin{abstract}
High-frequency broadband (200-300 kHz) acoustic scattering techniques have been used to observe the diffusive regime of double-diffusive convection in the laboratory. Pulse compression signal processing techniques allow 1) centimetre-scale interface thickness to be rapidly, remotely, and continuously measured, 2) the evolution, and ultimate merging, of multiple interfaces to be observed at high-resolution, and 3) convection cells within the surrounding mixed layers to be observed. The acoustically measured interface thickness, combined with knowledge of the slowly-varying temperatures within the surrounding layers, in turn allows the direct estimation of double-diffusive heat and buoyancy fluxes. The acoustically derived interface thickness, interfacial fluxes and migration rates are shown to support established theory. Acoustic techniques complement traditional laboratory sampling methods and provide enhanced capabilities for observing the diffusive regime of double-diffusion in the ocean.
\end{abstract}

Keywords Double-diffusion · Broadband acoustics · Convection

\section{Introduction}

A fluid that is stably stratified can undergo convection if the different components controlling the fluid density

T. Ross

Department of Oceanography, Dalhousie University, Halifax, NS B3H 4J1, Canada

E-mail: tetjana@dal.ca

A. Lavery

Applied Ocean Physics and Engineering, Woods Hole Oceanographic Institution,

Woods Hole, MA 02543, USA -diffuse at different rates. When fresh cold water overlies salty warm water, the temperature gradient has a destabilising effect on the density, which must be balanced by the salinity gradient. Because heat diffuses much faster than salt, however, these systems tend to a state where well-mixed convecting layers are separated by very thin double-diffusive interfaces (Turner, 1974; Linden and Shirtcliffe, 1978). In addition to their interest to fluid dynamists, double-diffusive interfaces are a ubiquitous, though difficult to sample, feature of polar oceans (Kelley et al, 2003).

The heat and salt fluxes associated with diffusive convection are the subject of debate in both oceanographic and laboratory studies. In the ocean, the discussion centres on whether diffusive convection is just an oddity or whether diffusive convection fluxes play a significant role in global ocean dynamics. The ocean is most susceptible to diffusive convection in polar regions where observations are limited due to remoteness and the difficulty of profiling in ice. Also, the doublediffusive interfaces that have been observed in these regions are typically at depths of 100 to 350 meters (Neal et al, 1969; Muench et al, 1990; Robertson et al, 1995) making profiling slow and thus making traditional microstructure or optical measurements very time consuming. This line of inquiry could therefore benefit greatly from a remote sensing technique that increases the rate and resolution at which data are collected. In the laboratory, the controversy centres on what is the appropriate theoretical form for the flux through the interface. The classical 4/3-heat-flux-law (Turner, 1965) is widely applied, though laboratory data do not support this power law for single component diffusion and there is little empirical evidence for the diffusive convection case (Kelley et al, 2003). One way to shed additional light on this debate is to obtain continuous measure- 
ments of the interface thickness, thereby allowing the continuous calculation of the conductive fluxes for comparison with theory.

Lavery and Ross (2007) showed that the sharp sound speed and density contrasts generated by double-diffusive interfaces scatter sound at observable levels and that interface thickness can be remotely and non-invasively measured using high-frequency broadband acoustic scattering techniques. This paper exploits those techniques in a series of laboratory experiments covering a wide range of interface parameters. It is shown that broadband acoustic scattering techniques complement traditional laboratory techniques, and have the potential of offering great advantages for studying double-diffusion in the ocean. In section 2 , the relevant details of the laboratory experiment and analysis techniques are described. In section 3, the acoustic backscattering observations are used to illuminate the migration and eventual merging of multiple double-diffusive interfaces and the acoustically determined migration rates are compared with theory. In section 4, interface thicknesses calculated using broadband acoustic techniques, micro-temperature data and optical shadowgraph observations are presented. In section 5 , it is demonstrated that broadband acoustic scattering techniques can result in direct estimates of fluxes through the interface and that the acoustically estimated interface thickness is in good agreement with theoretical predictions. In section 6 , it is argued that elevated acoustic scattering detected above the interface, most likely due to parcels of warm salty water from the bottom mixed layer infiltrating the top cold-fresh layer, and resulting in apparent "streaks" in the acoustic scattering returns, are actually acting as passive tracers of convection cells in the upper layer, thus allowing the convection cells to be imaged and their speeds inferred. The inferred speeds of the convection cells are compared with theoretical estimates based on the buoyancy flux through the interface (Deardorff, 1970).

\section{Laboratory set-up and methods}

\subsection{Laboratory set-up}

High-frequency broadband acoustic observations of doublediffusive interfaces were performed in a insulated cylindrical tank (470 $\mathrm{cm}$ deep and $91.4 \mathrm{~cm}$ in diameter) (Schmitt et al, 2005; Lavery and Ross, 2007). Single double-diffusive interfaces were formed by filling the bottom of the tank with salt water, then very slowly floating fresh water on top through a sponge. The salty layer was then heated from below and the fresh water cooled from above to create and maintain a sharp interface. The temperature and salinity at one point in each well-mixed layer were monitored continuously with calibrated Falmouth Scientific, Inc. conductivity and temperature (CT) sensors fixed to the tank wall at depths of 100 and $300 \mathrm{~cm}$. High-resolution vertical temperature and conductivity profiles across the interface were measured at intervals by traversing a Precision Measurement Engineering, Inc. (PME) conductivity and temperature microstructure sensor at $10 \mathrm{~cm} / \mathrm{s}$ through the depth of the tank. This fall rate was chosen to ensure that the profiles were not biased by the variability of the interface, as high ping-rate acoustic observations of the interface showed that the scattering from the interface decorrelates in around 2 seconds (Lavery and Ross, 2007). These profiles were used to calculate salinity profiles. Two windows set into opposite sides of the tank at mid-depth allowed the interfaces to be observed using shadowgraph techniques.

A typical experiment lasted 2-3 weeks until the stabilising salinity step $(\Delta S)$ eroded to the point that the destabilising temperature differential $(\Delta T)$ could no longer be maintained and the interface overturned. Varying the amount of forcing (i.e. cooling and heating applied at the top and bottom), allowed control of $\Delta T$ and subsequently of the density ratio, $R_{\rho}=$ $(\beta \Delta S) /(\alpha \Delta T)$, where $\alpha$ and $\beta$ are the coefficients of thermal expansion and haline compression of salt water. Varying $\Delta T$, combined with the slow but steady diminishment of $\Delta S$, allowed $R_{\rho}$ to be varied over a wide range during the course of the experiments. $R_{\rho}$ is the primary parameter determining the stability and nature of the double diffusive interface. Gravitational instability occurs once $R_{\rho}$ falls below 1 .

The majority of the experiments were set-up with a single double-diffusive interface, as described above. Detailed results from two single-interface experiments are presented here, and are representative of all the single interface experiments performed. If the tank was filled sloppily or the interface was mixed, creating a gradient extending over $\sim 20 \mathrm{~cm}$ between the layers, multiple sharp interfaces would appear once the heating and cooling was turned on. These interfaces would migrate and ultimately merge into one interface. Results of an experiment involving multiple interfaces are discussed in detail in section 3 .

\subsection{Acoustic data and analysis}

All the acoustic data presented here were collected with a pair of $250 \mathrm{kHz}$ broadband transducers, with approximately octave wide bandwidth, driven with a "chirp" transmit pulse that linearly increased in frequency from 
a start frequency, $f_{i}$ (typically $200 \mathrm{kHz}$ ), to an end frequency, $f_{f}$ (typically $300 \mathrm{kHz}$ ), for the duration of each pulse (typically $200 \mu \mathrm{s}$ ). The ping rate was usually 1 Hz. The bandwidth, pulse duration and ping rate were altered occasionally, as indicated in the figure captions. The transducers were placed adjacent to each other in order to collect acoustic backscattering echoes from the double-diffusive interface and were typically focused at a range of $75 \mathrm{~cm}$. When imaging a single interface, the transducers were placed $75 \mathrm{~cm}$ above the interface, that is, at a range, $r=75 \mathrm{~cm}$. Acoustic data were collected in 30-minute intervals (typically resulting in 1800 individual ensonifications or pings) various times a day for the duration of the experiments. See (Lavery and Ross, 2007) for a full description of the experimental set-up, including the transducers and the pulse-echo system.

A useful technique commonly used with broadband acoustic data is pulse compression (Chu and Stanton, 1998; Lavery and Ross, 2007; Stanton and Chu, 2008). The compressed pulse $(C P)$ output is a scaled crosscorrelation of the received signal with a calibration signal,

$C P(t)=k_{C P}^{-1} v^{R}(t) \star v_{\text {cal }}^{R}(t)$,

where $v^{R}(t)$ is the received voltage time series from the double-diffusive interface, $v_{c a l}^{R}(t)$ is the received calibration pulse, collected with the transmit and receive transducers facing each other, and $\star$ indicates a crosscorrelation. The parameter $k_{C P}$ is the auto-correlation function of $v_{\text {cal }}^{R}$ evaluated at zero lag. The envelope of the compressed-pulse output, $E_{C P}$, is a good proxy for scattering intensity across the whole bandwidth and benefits from increased signal-to-noise ratio and temporal resolution (Chu and Stanton, 1998; Stanton and Chu, 2008).

There was great variability in the acoustic returns from the double-diffusive interface. The overall amplitude, the spectral structure and the number of distinct returns in the compressed pulse output were all highly variable (for details see Lavery and Ross, 2007). Lavery and Ross successfully model the scattering from the interface as a weakly-scattering, inhomogeneous, multilayered medium (i.e. scattering from multiple sharp interfaces separated by thin finite sublayers with homogeneous sound speed and density). In this framework, profiles with sharp gradients in density and sound speed at both the top and bottom of the interfacial region will lead to elevated scattering from these high-gradient regions, thereby revealing the top and bottom of the interface.

\subsubsection{Interface thickness from acoustic measurements}

The double-diffusive interfaces were typically several centimetres thick, which is significantly smaller than the volume ensonified acoustically. Making use of the enhanced resolution achieved using pulse compression techniques it was possible to isolate returns that had exactly two peaks in the $E_{C P}$ time series. These returns are consistent with scattering from the top and the bottom of the interface (Lavery and Ross, 2007). The remotely inferred interface thickness $\Delta z_{a c}$, is thus given by,

$\Delta z_{a c}=c /(2 \Delta t)$,

where $\Delta t$ is the time between the arrivals of the first and second peaks in the $E_{C P}$ time series. This method is illustrated in panel a of Fig. 1.

\subsection{Microstructure data and analysis}

The PME micro-temperature and conductivity data, digitised at $1 \mathrm{kHz}$, were first filtered with a Butterworth filter with a 100-Hz cut-off. Each micro-conductivity profile was calibrated using the fixed sensor data (assuming a linear response between the calibration points in the upper and lower layers). The micro-temperature probes, calibrated by the manufacturer, were regularly compared with the more accurate fixed CT sensors and re-calibrated if there was significant disagreement. Before calculating salinity, density and sound speed profiles (Fofofnoff and R.C. Millard, 1983), the conductivity data were filtered to match the response of the temperature sensors (following the method outlined in (Schmitt et al, 2005)).

These profiles showed very little variability in the temperature and conductivity in the layers surrounding the interface, confirming that the CT sensor measurements are representative of these well-mixed layers. At times, however, weak variability reaching 10 to 30 centimetres above the interface was observed, which are likely related to the "streaks" of backscatter observed in the acoustic signals (section 6).

\subsubsection{Interface thickness from micro-temperature measurements}

Interface thicknesses were also extracted from the PME temperature data by peforming a least-squares fit between the measured temperature profiles and an idealised hyperbolic tangent function,

$T=T_{0}+\frac{\Delta T}{2} \tanh \left(\frac{z-z_{0}}{L}\right)$, 

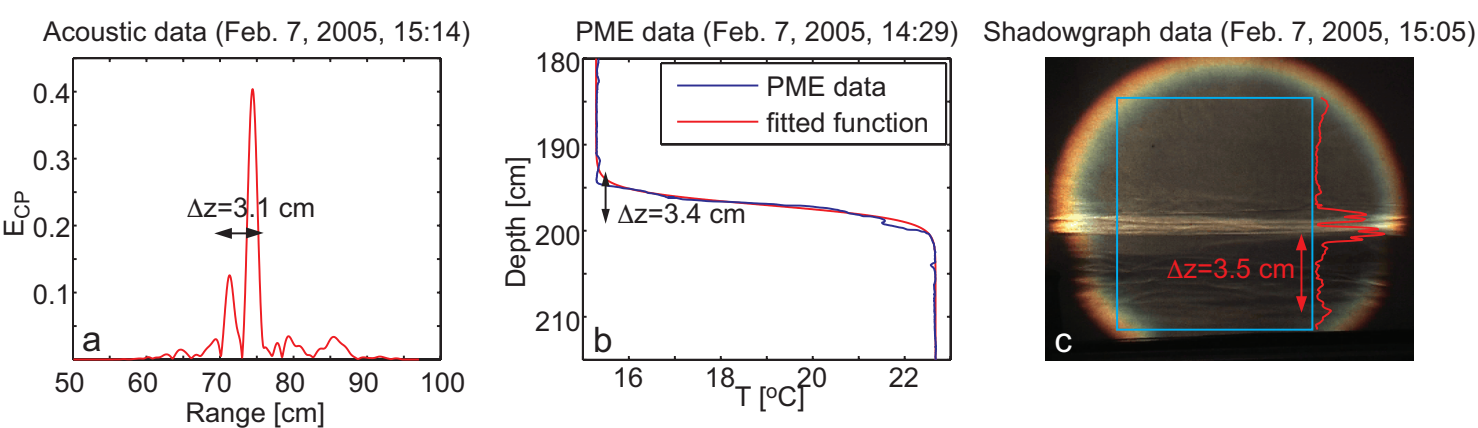

Fig. 1 Panels $\mathbf{a}, \mathbf{b}$ and $\mathbf{c}$ illustrate how interface thicknesses were estimated from the acoustic data (from (2)), PME profiles (i.e. $\Delta z_{p m e}=2 L$ from (3)), and shadowgraph data, respectively. The blue box in panel e shows an example of the windowing applied to the shadowgraph images to remove the dark areas outside the collimated beam and the red line shows the absolute value of the gradient of the light intensity summed across the box.

where $\mathrm{z}$ is depth, $z_{0}$ is the depth of the interface and $T_{0}$ is the mean of the temperatures in the surrounding layers. The interface thickness is given by $\Delta z_{p m e}=2 L$, which is illustrated in panel $\mathbf{b}$ of Fig. 1. Although the goal was to determine the interface thickness (i.e. $L$ ), $\Delta T, T_{0}$ and $z_{0}$ were all left as free parameters in the fitting procedure. As a check on the accuracy of the fits, $T_{0}-\Delta T$ and $T_{0}+\Delta T$ were compared with the upper and lower CT sensors measurements for single-interface profiles and were always within the $0.05^{\circ} \mathrm{C}$ accuracy of the CT sensors.

\subsection{Shadowgraph imagery and analysis}

Optical shadowgraph data were collected by projecting a bright, collimated beam of light horizontally across the centre of the tank, through the double-diffusive interface, and onto tracing paper. The variations in the index of refraction of light due the interface refracted the light in the interfacial region, allowing the interface to be imaged. These images were recorded at a rate of $15 \mathrm{~Hz}$ using a digital video camera.

\subsubsection{Interface thickness from shadowgraph imagery}

Like the acoustic data, the shadowgraph images often showed a distinct top and bottom to the light refracted by the interface. The digital video was analysed frame by frame. Each frame was windowed to exclude dark regions around the collimated beam (see panel $\mathbf{c}$ of Fig. 1), the light intensity was summed across the image to give a single vertical profile and an interface thickness, $\Delta z_{s g}$, was extracted from each image showing two distinct peaks in the absolute gradient of this light intensity profile. An example of the absolute gradient of the light intensity profile is shown in panel c of Fig. 1; note that both the bright top band and the bottom of the dark band produce peaks. Because the top of the interface was much brighter than the bottom, peaks occurring above the maximum in the absolute gradient of the light intensity were neglected. As discussed in Lavery and Ross (2007), most images with more than two distinct peaks (excluding any above the maximum) appeared turbulent and were rejected from the analysis.

\subsection{Fluxes across the interfaces}

The buoyancy fluxes $(J)$ across the interfaces were estimated from the standard conductive buoyancy flux equation

$J=\left(1-R_{F}\right) g \alpha \kappa_{T} \frac{d T}{d z} \approx\left(1-R_{F}\right) g \alpha \kappa_{T} \frac{\Delta T}{\Delta z}$,

where $R_{F}$ is the flux ratio between salt and heat (around .15 for the high density ratios of this experiment (Huppert, 1971; Kelley, 1990)), $g$ is gravitational acceleration, $\alpha$ and $\kappa_{T}$ are the coefficient of thermal expansion and the thermal diffusivity of the water, and $d T / d z$ is the temperature gradient across the interface, which was approximated by the ratio of the temperature step to the interface thickness (either $\Delta z_{p m e}$ or $\Delta z_{a c}$ as appropriate).

The heat fluxes (in Watts) across the interfaces are simply related to the buoyancy fluxes by

$Q=\frac{A C_{p} \rho}{g \alpha\left(1-R_{F}\right)} J$

where $A=.66 \mathrm{~m}^{2}$ is the cross-sectional area of the tank, $C_{p}$ is the specific heat of the water and $\rho$ is the mean of the water densities in the surrounding layers.

At the bottom and top of the tank, (4) cannot be applied. The fluxes through these solid boundaries were estimated using the approximate temperature difference between the boundary (in one case the piping of the chiller, in the other, the heating pad) and the water 
in the adjacent layer $\left(\Delta T_{1 / 2}\right)$, and the formulation for buoyancy fluxes for a single plate (Turner, 1973),

$J=\frac{(0.089)\left(2^{4 / 3}\right)}{A}\left(1-R_{F}\right)\left(\frac{\kappa_{T}^{2}}{\nu}\right)^{1 / 3}\left(g \alpha \Delta T_{1 / 2}\right)^{4 / 3}$

where $\nu$ is the kinematic viscosity of the water. These boundary fluxes are approximate. The temperatures that either the heater or chiller were set to were noted throughout the experiments, but no direct measurements of these temperatures were made.

Previous measurements of the heat supplied to the tank through the bottom boundary showed $Q \approx 400 \mathrm{~W}$ (Schmitt et al, 2005). Inverting (5), this would give $J \approx 3$ to $4 \times 10^{-7} \mathrm{~m}^{2} / \mathrm{s}^{3}$. Although no direct measurements were made during these experiments, assuming that as the same equipment was used, the power supplied was of the same order, this can be used as a first-order check on the buoyancy fluxes through bottom boundary calculated from (6). The buoyancy fluxes from (6) were $J \approx 1$ to $6 \times 10^{-7} \mathrm{~m}^{2} / \mathrm{s}^{3}$.

\section{Evolution of multiple interfaces and inferred migration rates}

One of the most striking images emerging from these laboratory experiments was the temporal evolution of multiple double-diffusive interfaces merging into one (Fig. 2). The series of interfaces pictured in Fig. 2 formed after the forcing was applied to initial temperature and salinity profiles that had well-mixed upper and lower layers separated by an approximately linear temperature and salinity gradient extending $60 \mathrm{cms}$. The sequence starts 12 days after the forcing was first applied.

All coloured panels in Fig. 2 show $\log _{10}\left(E_{C P}\right)$ as a proxy for scattered intensity. The PME temperature profiles show sharp temperature steps associated with each of the scattering layers. Not all of the acoustic pings are plotted in Fig. 2, depending on the ping rate used, 30 to 120 pings were averaged to give a regular 8 minute spacing to the acoustic data.

Unlike the PME profiles, the acoustic data allow the double-diffusive interfaces to be remotely tracked over long periods without disrupting them. This results in an independent estimate of the interface migration speed, which could not be achieved with the PME data without resorting to theory. Interface migration speeds were estimated from the acoustic data by locating the peaks in the 8-minute averaged $C P$ (editing out the known side-lobes and the shadowgraph window reflections) and then fitting a straight line to the data surrounding the time of interest, usually a time window of about 3 hours, but up to 5 hours if there were gaps in the data. The slope of this line is the local migration rate. These acoustically determined migration rates are plotted in Fig. 3 for the times of the PME profiles shown in Fig. 2. The error bars show bootstrapped 95\% confidence intervals (Efron and Gong, 1983).

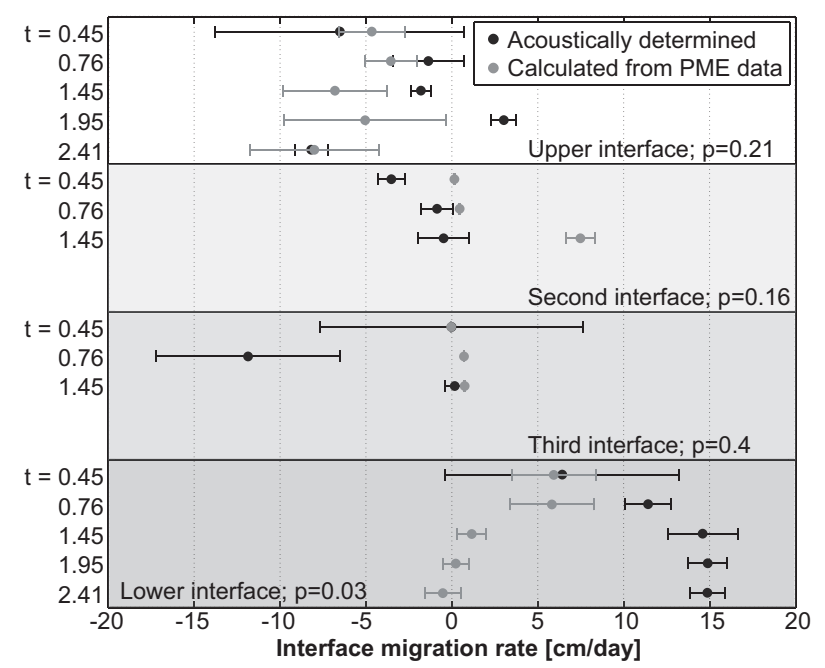

Fig. 3 A comparison of acoustically determined interface migration rates with those calculated from (7) using the PME data shown in Fig. 2. Positive values indicate upward migration. The probability ( $p$, estimated from a Student t-test) that the migration rates calculated by the two methods agree is shown for each interface.

Differences in buoyancy flux from one layer to the next can cause the interface between them to become unstable (Huppert, 1971) and migrate (Kelley, 1987; McDougall, 1981). The layer with the higher buoyancy flux will have more active convection and erode the adjacent layer, causing the interface to move into that layer. The migration speed, $e$, can be expressed as (Kelley, 1987)

$e=\frac{0.2\left(j_{2}-j_{1}\right)}{g \Delta \rho / \rho}$

where $j_{1}$ and $j_{2}$ are the buoyancy fluxes in the layer above and below the interface, respectively, and $\Delta \rho$ is the density difference across the interface. Using (7), the interface migration speeds for the times of the PME profiles in Fig. 2 were calculated. The buoyancy fluxes in the layers are taken to be the average of the conductive buoyancy fluxes across its two boundaries; i.e. $j_{1}=\left(J_{0}+J_{1}\right) / 2$ where $J_{0}$ is the flux through the surface (from (6)) and $J_{1}$ is the flux through the first interface (from (4) with $\Delta z=\Delta z_{p m e}$ ).

Fig. 3 compares the acoustically derived interface migration speeds to those calculated from the PME data using (7). The uncertainties in the calculated migration rates for the upper and lower interfaces are dominated by the large uncertainties assigned to the 


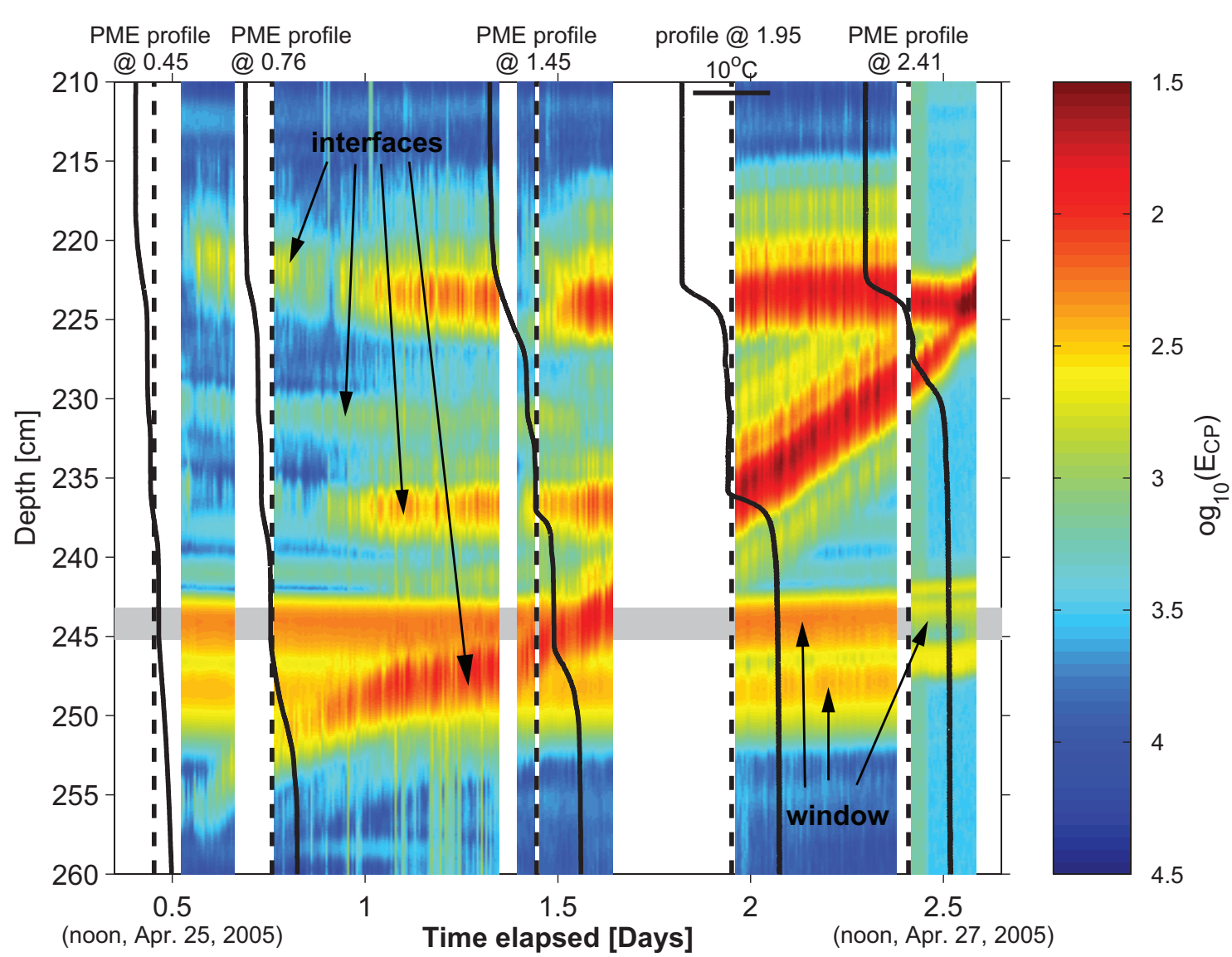

Fig. 2 False colour plot of pulse-compressed acoustic returns from a series of double-diffusive interfaces in the tank. The data were collected using a reduced bandwidth of $225-275 \mathrm{kHz}$ and a $100 \mu \mathrm{s}$ pulse duration in all panels except the last, which is $250 \mathrm{kHz}$ narrowband and has a pulse duration of $5.5 \mu \mathrm{s}$ (which is why it has a different appearance). Temperature profiles collected with the PME sensor are superimposed on the acoustic data. The grey bar indicates the approximate range to the edges of the windows cut into the side of the tank to allow shadowgraph imagery, the steady reflections from which can be seen between 245 and $250 \mathrm{~cm}$ in the acoustic data.

estimates of the fluxes through the top and bottom of the tank, while the uncertainties in the second and third interface migration rates reflect the relatively small uncertainties (standard deviations) associated with fitting (3) to the PME profiles. The uncertainties in the acoustic estimate for the first profile $(t=0.45)$ may be underestimated, because the acoustic data had to be extrapolated (as there were no acoustic data collected before the first PME profile in Fig. 2). To compare the agreement of the migration rates calculated by these two methods for each interface, a simple (unweighted), paired Student t-test was applied. The upper three interfaces showed agreement between the methods at probabilities well above the $5 \%$ level $(21 \%, 16 \%$ and $40 \%$, respectively). Only the lower interface showed significant disagreement, where (aside from the first profile) the PME estimate is consistently 2 to 3 times smaller than what was observed. This could be because the buoyancy flux through the bottom of the tank has been underestimated, although estimating the flux from the four observed migration rates that disagree leads to heat fluxes 2 to 3 times those measured previously $(Q=800$ to $1100 \mathrm{~W}$ as compared with $400 \mathrm{~W}$ from Schmitt et al (2005)).

\section{Comparison of interface thickness measurements}

The interface thicknesses from all three methods are shown in Fig. 4 . While there is some inconsistency in using a smooth hyperbolic function to deduce the interface thickness for the PME data while using a two edge model for both the shadowgraph and acoustic data, the resulting comparison shows good agreement. The tanh function was chosen as it is the simplest functional form consistent with the theoretical expressions of Linden and Shirtcliffe (1978) and should result in only one edge within the interface. The measured PME profiles rarely followed this form exactly. Generally, the profiles showed a sharp upper step and, only occasionally, some 
variability near the end of the discontinuity that could possibly cause a second source of optical or acoustical scattering. Both the shadowgraph and acoustic data, however, showed many instances of two edges. For instance, as many as $80 \%$ of acoustic observations showed two edges in the the Feb. 2005 experiment (Fig. 4). In the end, while they are are based on inconsistent views of the details of the interface, what is important is that the three methods produce very similar results.

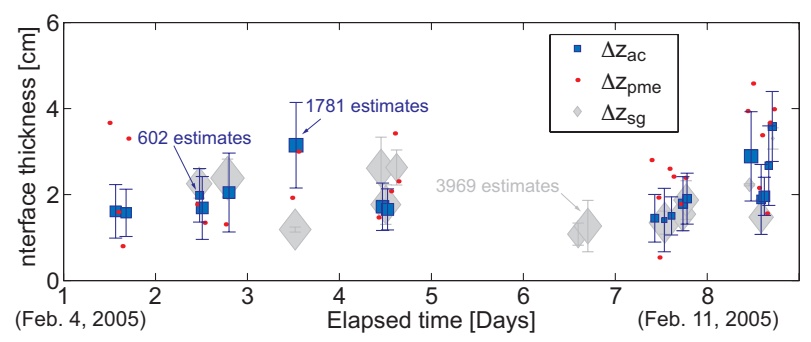

Fig. 4 Plot showing a comparison of interface thicknesses estimated from the acoustic data (blue squares, illustrated in panel a of Fig. 1), individual PME profiles (red dots, illustrated in panel b of Fig. 1), and shadowgraph data (grey diamonds, illustrated in panel $\mathbf{c}$ of Fig. 1), respectively. The squares and diamonds are scaled by the number of estimates that went into each average. The errorbars indicate the standard deviation. The acoustic collection periods were all 30 minutes (i.e. 1800 pings) long. The collection period for the shadowgraph video was approximately 9.5 minutes (i.e. around 8500 frames were analysed for each diamond plotted).

There is, however, significantly more variability in the PME derived interface thickness because no averaging was possible. It was impossible to do repeat PME profiles and collect a distribution of $\Delta z_{p m e}$, because each profile of the PME sensors through the interface disturbed it, causing erratic, large-amplitude oscillations of the interface echoes which took about 15 minutes to diminish to pre-profile levels. However, the PME derived interface thickness generally fell within the distribution of the $\Delta z_{a c}$ and the $\Delta z_{s g}$, indicating that they are likely sampling the same distributions. As the PME data were sparse (the micro-temperature derived interface thicknesses were outnumbered by acoustic observations of interface thickness by a ratio of around 150:1), it is clear that the 30-minute mean acoustically estimated interface thickness is a more accurate representation of the mean interface thickness on timescales of hours than the instantaneous shapshot of $\Delta z_{p m e}$ that results from one or two profiles.

\section{Acoustically estimated heat flux and density ratio}

The fact that broadband acoustics provides an estimate of interface thickness, combined with the fixed CT sensor data, allowed the calculation of conductive heat fluxes across a single interface without the need for micro-temperature data. The heat flux estimated from (4) and (5) for the two long-term experiments on a single double-diffusive interface are plotted as grey circles in the upper panels of Fig. 5. In this application of (4) and (5), $\Delta T$ is the difference in the CT sensor measurements in the upper and lower layers and $\Delta z=\Delta z_{a c}$ is the average of all the acoustically determined interface thicknesses for pings with exactly two interfacial $E_{C P^{-}}$ peaks during a 30-minute section of acoustic data.

Another option for estimating the heat flux through the interface, based on the 4/3 flux law (Kelley, 1990),

$J=0.0032\left(1-R_{F}\right) e^{\frac{4.8}{R_{\rho}^{0.72}}}\left(\frac{\kappa_{T}^{2}}{\nu}\right)^{1 / 3}(g \alpha \Delta T)^{4 / 3}$,

can be applied without knowledge of the interface thickness, though it does require knowledge of the density ratio of the interface. The two methods agree fairly well, as long as $R_{\rho}>3$ (Fig. 5). For $R_{\rho}<3$, the fluxes calculated using the $4 / 3$ law are unrealistically high, likely because at these low $R_{\rho}$ the interface is more susceptible to disruptions caused by convection in the surrounding layers and thereby violating the assumptions necessary to derive (8) (Worster, 2004). Additionally, recent work has called into question the validity of applying a constant flux law (Worster, 2004) because the timedependence of the fluxes and sensitivity to initial conditions are ignored by formulations such as (8). Thus, the acoustic technique allows a way of remotely and possibly more accurate monitoring of heat flux across the interface as it reaches the point of instability (and $R_{\rho}$ tends to one).

The bottom panels of Fig. 5 show the density ratio (calculated from the CT sensor data), along with the acoustically measured interface thickness. Note that the interface thickness and density ratio are correlated; something predicted by the theory of Linden and Shirtcliffe (1978), and modified by Worster (2004) to

$\Delta z_{L S}=\frac{1}{\lambda(\tau)}\left[\frac{16 \nu \kappa_{T}\left(1-\tau^{1 / 2}\right)^{4}}{g \alpha \Delta T\left(1-\tau^{1 / 2} R_{\rho}\right)^{4}}\right]^{1 / 3}$,

where $\tau$ is the diffusivity ratio $\left(\frac{\kappa_{S}}{\kappa_{T}} ; \kappa_{S}\right.$ is the diffusivity of salt) and $\lambda(\tau)$ is a parameter that Worster (2004) argues can be treated as an empirical constant to account for sensitivity to initial conditions in different experimental set-ups. Worster (2004) found that $\lambda(\tau)=0.075$ gave the best agreement between predicted heat fluxes 

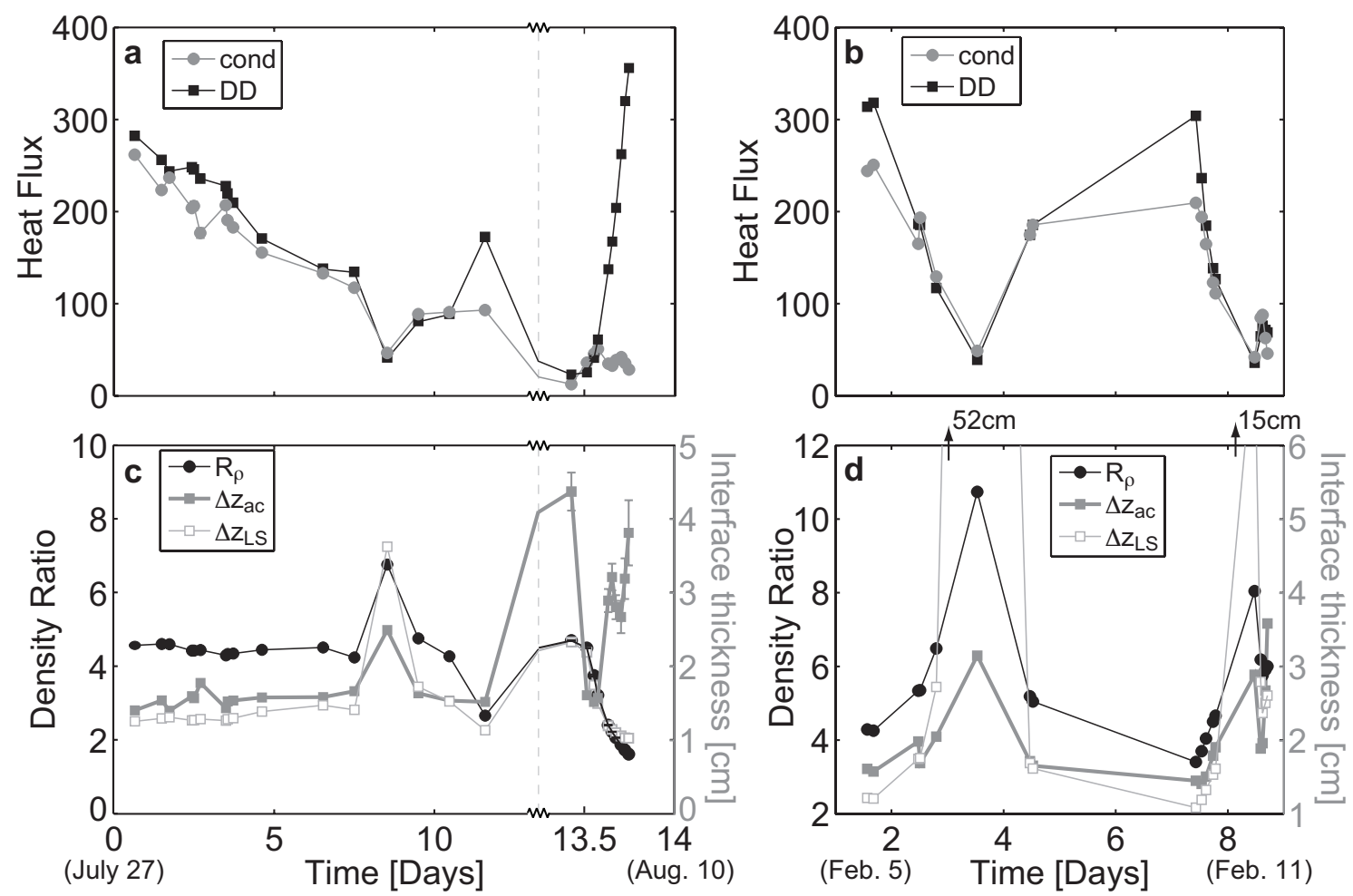

Fig. 5 Panels $\mathbf{a}$ and $\mathbf{b}$ show plots of heat flux estimated from (5) with (4), labelled cond, and with (8), labelled DD, for two experiments; July and August 2004 (a) and February 2005 (b). Panels c and d show plots of density ratio, R $\rho$, and interface thickness, both measured, $\Delta z_{a c}$, and estimated from (9), $\Delta z_{L S}$, for the same time periods. Each symbol corresponds to an average over a 30-minute acoustic file and the errorbars are the standard error (most errorbars are smaller than the symbol). All the acoustic data used were collected with the full $200-300 \mathrm{kHz}$ bandwidth, $200 \mu$ s pulse duration and $1 \mathrm{~Hz}$ pulse rate.

and those observed by Turner (1965) with a set-up similar to this one. In Fig. $5, \Delta z_{L S}$ are plotted for $\lambda(\tau)=0.075$. However, it is $\lambda(\tau)=0.091$ that minimised the mean square difference between $\Delta z_{a c}$ and $\Delta z_{L S}$ for this experiment. Note that (9) has a singularity at $\tau^{1 / 2} R_{\rho}=1$, which explains the very large values of $\Delta z_{L S}$ in the bottom panels of Fig. 5 .

Interface thickness also depends on $\Delta T$ in (9), but assuming that $\Delta S$ doesn't change quickly (due to the much smaller diffusivity of salt) $\Delta T=\beta \Delta S / \alpha R_{\rho}$ is proportional to $1 / R_{\rho}$ and $\Delta z$ is proportional to $\left(R_{\rho} /(1-\right.$ $\left.\left.\tau^{1 / 2} R_{\rho}\right)^{4}\right)^{1 / 3}$. Thus, changes in the acoustically measured interface thickness could be used as a proxy for changes the density ratio in field studies where other data are unavailable.

\section{Convection}

Broadband acoustics also allowed the observation of the convection above the double-diffusive interface, which provides an acoustic analogy to tracer release observations of convective cells and allows the compilation of a distribution of the speeds of the convective cells. Fig. 6 shows two consecutive acoustic data collection runs, each lasting 30 minutes and separated by about 20 minutes. Again, the logarithm of the envelope of the compressed pulse output is plotted in false colour as a function of time and depth in the tank, but there is no averaging of pings (these pings had the full $200-300 \mathrm{kHz}$ bandwidth, $200 \mu$ s pulse length and $1 \mathrm{~Hz}$ ping rate). There is much ping-to-ping variability; both in the intensity (upper panel) and the acoustic estimate of the interface thickness (bottom panel). This variability is discussed at length in (Lavery and Ross, 2007) and is likely due to interface displacements caused by internal waves that are excited by convection in the layers. In addition, artefacts due to the pulse compression signal processing side-lobes (Chu and Stanton, 1998) are visible, spanning approximately $10 \mathrm{~cm}$ on either side of the interface. Weak scattering from the top of the shadowgraph windows can also be seen at a depth of 1.81 m. Many "streaks" of elevated acoustic scatter above the interface (particularly in the first section of data) stand out above this background. An estimate of the expected speed of the convective cells in the layer is given by (Deardorff, 1970),

$w_{*}=(-J H)^{1 / 3}$, 
where $H$ is the layer thickness $(\sim 2 \mathrm{~m})$ and $J$ is the buoyancy flux from (4) with $\Delta z=\Delta z_{a c}$. Thus, $w_{*} \sim 0.5$ $\mathrm{cm} / \mathrm{s}$ for the first section of data. The distribution of rise/fall speeds of the "streaks" for this section of data is shown in Fig. 6. Both normal $(\mu=0.35, \sigma=0.22$ $\mathrm{cm} / \mathrm{s})$ and $\log$-normal $(\ln \mu=0.29 \mathrm{~cm} / \mathrm{s})$ distributions are plotted for reference, though neither is statistically significant. However, the log-normal distribution does a far better job of representing the data when the four "streaks" with very fast rise speeds of 2.4 to $9 \mathrm{~cm} / \mathrm{s}$, omitted from the histogram, are included in the fit.

The most likely explanation for these "streaks" is that they are small parcels of water with contrasting density and sound speed created by the convection. As they are swept along the convective path, they mix with the surrounding water creating density and sound speed microstructure which scatters sound (Lavery et al, 2003; Ross et al, 2004). Regardless of the origin of the scattering or the form of the distribution, the elevated scattering above the interface appears to be a passive tracer of the convective cells. There is good agreement between the mean of the distribution of the "streak" speeds, $w \sim 0.35 \pm 0.22 \mathrm{~cm} / \mathrm{s}$, and the estimate based on the buoyancy flux formulation, (10), $w_{*} \sim 0.5 \mathrm{~cm} / \mathrm{s}$.

At the beginning of the second section of acoustic data plotted in Fig. 6 (at the time marked by an arrow) the external forcing (both the heater and chiller) were shut off. Within ten minutes, the "streaks" disappear, which supports the assumption that the scattering is from microstructure. As the convection slows convective parcels of water would be created more infrequently or have a weaker contrast with the surrounding water as the convection weakened. Acoustically, this would mean fewer and weaker scattering from the convective parcels until they become too weak to be seen above the background.

During the weakening of the convection, the ping to ping coherence also increased. In Fig. 6 this can be seen in both the intensity and the interface thickness data. Additionally, pings that show two clear $C P$-peaks appear more consistently without the strong external forcing, which translates into more frequent interface thickness estimates (coloured boxes at the bottom of the bottom panel enumerate how many interface thickness estimates were made for each 5 minutes of data). Again, this supports the idea that the variability of the returns from the interface are caused by convective pulses periodically disturbing the interface and causing internal waves. As the strong externally forced convection dies down, so do the disturbances.

\section{Conclusions}

Acoustic observations of double-diffusive convection in the laboratory not only provide elegant images of the migration and merging of interfaces and of convective motions in the surrounding layers, but also quantitative measures of interface migration rates, interface thicknesses and speeds of convective motions that compare favourably with theory. Additionally, acoustically determined interface thickness allowed direct estimates of fluxes through the interface, which is particularly relevant for $R_{\rho}<3$ as this is where the $4 / 3$ flux law formulation appears to break down, thus necessitating knowledge of the interface thickness to accurately estimate fluxes. This may prove a boon to field studies of double-diffusive convection, where $R_{\rho}$ if often less than 3 and data, particularly microstructure data, are often sparse. Microstructure data are sparse both because sampling opportunities are more limited in remote polar regions, but also, and perhaps more importantly, because profiling microstructure sensors the hundreds of meters to the depths of double-diffusive interfaces takes tens of minutes. Compare this with the tenth of a second it takes to make an acoustic profile and one can easily see how acoustics can fill the gaps between sparse microstructure profiles, perhaps leading to a better understanding of the underlying physics. Furthermore, the use of broadband acoustic backscattering techniques to estimate interface thickness could result in remote heat flux estimates, dramatically increasing the number of double-diffusive heat flux estimates which could lead to the resolution of the debate over whether they have oceanographic significance. Additionally, the remotely sensed interface thickness may be used as a proxy for density ratio.

Acknowledgements We thank Ray Schmitt for the use of his tank, Dan Kelley for useful comments on the manuscript, and the Doherty Foundation for funding Tetjana's postdoctoral scholarship at WHOI. Funding for this project was provided by the Ocean Acoustics program at the Office of Naval Research, and by the WHOI Cecil and Ida Greene Technology Award.

\section{References}

Chu D, Stanton T (1998) Application of pulse compression techniques to broadband acoustic scattering by live individual zooplankton. J Acoust Soc Am 104:39-55

Deardorff JW (1970) Convective velocity and temperature scales for the unstable planetary boundary layer and for rayleigh convection. J Atmos Sci 27:12111213 


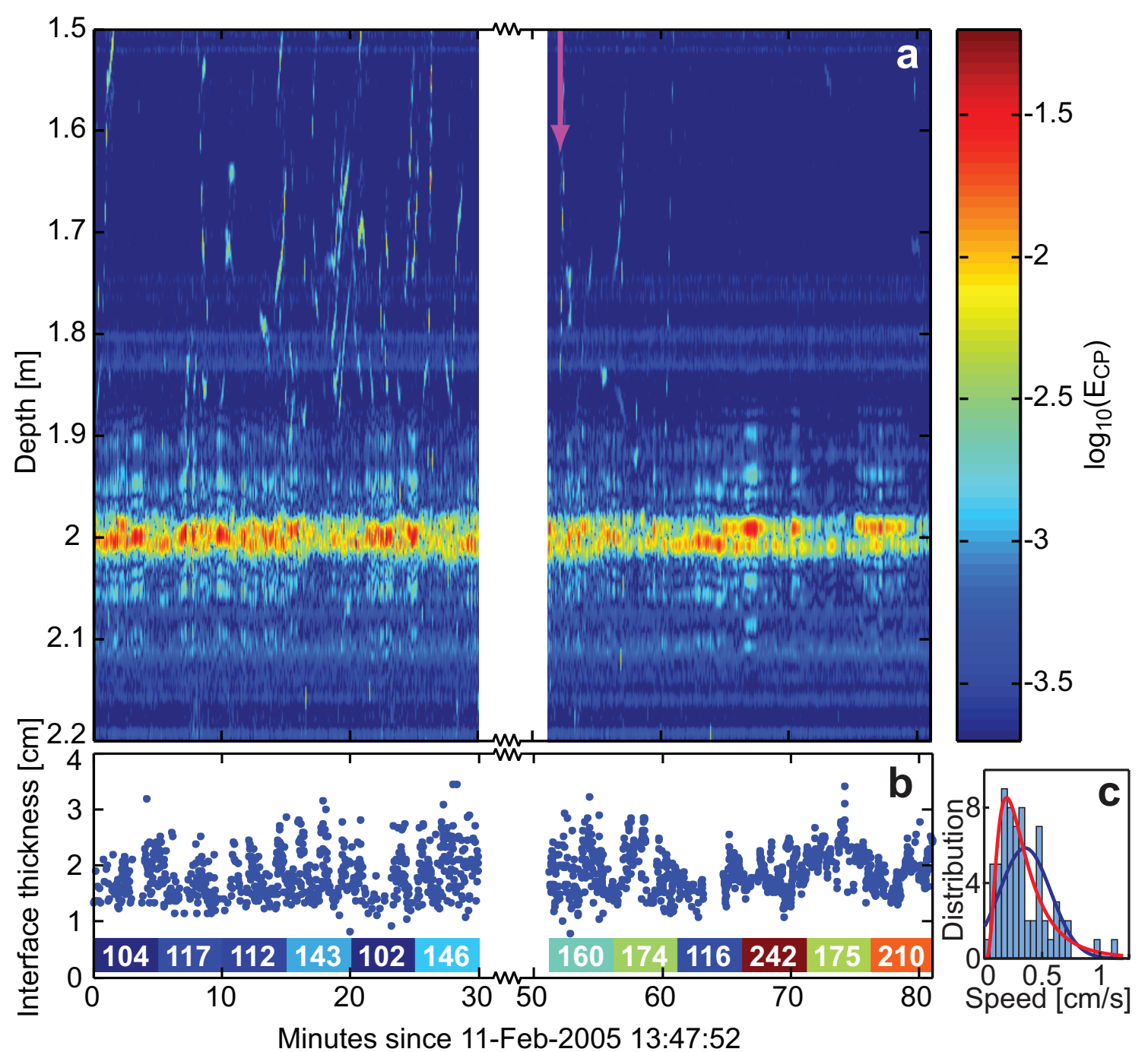

Fig. 6 Panel a: False colour plot of pulse-compressed 200-300 kHz acoustic returns from a single double-diffusive interface. At the time indicated by the arrow, the forcing (both active heating and cooling) was shut-off. Panel $\mathbf{b}$ : Interface thickness estimated from spacing between pairs of peaks in the pulse-compressed acoustic data in upper panel. The coloured boxes below the plotted data show the number of instances in that time period when there were exactly two peaks in the compressed pulse output (i.e. when an interface thickness could be measured). Panel c: Histogram of speeds of convective "streaks" for first section of data in panel a and the best fitting normal (blue) and log-normal (red) distributions.

Efron B, Gong G (1983) A leisurely look at the bootstrap, the jacknife, and cross-validation. The American Statistician 37:36-48

Fofofnoff N, RC Millard J (1983) Algorithms for computation of fundamental properties of seawater. Tech. rep., UNESCO, Paris

Huppert HE (1971) On the stability of a series of double-diffusive layers. Deep-Sea Res 18:1005-1021

Kelley D (1987) Interface migration in thermohaline staircases. J Phys Oceanogr 17:1633-1639

Kelley D (1990) Fluxes through diffusive staircases, a new formulation. J Geophys Res 95:3365-3371

Kelley D, Fernando H, Gargett A, Tanny J, Ozsoy E (2003) The diffusive regime of double-diffusive con- vection. Prog Oceanog 56:461-481

Lavery A, Ross T (2007) Acoustic scattering from double-diffusive microstructure. J Acoust Soc Am 122(3):1449-1462

Lavery AC, Schmitt RW, Stanton TK (2003) Highfrequency acoustic scattering from turbulent oceanic microstructure: the importance of density fluctuations. J Acoust Soc Am 114:2685-2697

Linden PF, Shirtcliffe TGL (1978) The diffusive interface in double-diffusive convection. J Fluid Mech $87: 417-432$

McDougall TJ (1981) Double-diffusive convection with a nonlinear equation of state, Part II. Laboratory experiments and their interpretation. Prog Oceanogr 
10:91-121

Muench RD, Fernando HJS, Stegen GR (1990) Temperature and salinity staircases in the Northwestern Weddell Sea. J Phys Oceanogr 20:295-306

Neal VT, Neshyba S, Denner W (1969) Thermal stratification in the Arctic Ocean. Science 166:373-374

Robertson R, Padman L, Levine MD (1995) Fine structure, microstructure, and vertical mixing processes int he upper ocean in the western Weddell Sea. J Geophys Res 100(C9):18,517-18,535

Ross T, Garrett C, Lueck R (2004) On the turbulent co-spectrum of two scalars and its effect on acoustic scattering from oceanic turbulence. J Fluid Mech 514:107-119

Schmitt RW, Millard RC, Toole JM, Wellwood WD (2005) A double-diffusive interface tank for dynamicresponse studies. J Mar Res 63:263-289

Stanton TK, Chu D (2008) Calibration of broadband active acoustic systems using a single standard spherical target. J Acous Soc Am 124:128-136

Turner JS (1965) The coupled turbulent transports of salt and heat across a sharp density interface. Intl J Heat Mass Transfer 8:759-767

Turner JS (1973) Buoyancy Effects in Fluids. Cambridge University Press

Turner JS (1974) Double-diffusive phenomena. Ann Rev Fluid Mech 6:37-55

Worster MG (2004) Time-dependent fluxes across double-diffusive interfaces. J Fluid Mech 505:287-307 